\section{PERCEPTION AND ACTION}

\section{Going With the Optic Flow}

BRUGgEMAN \& WARREN (2010). The direction of walking - but not throwing or kicking - is adapted by optic flow. Psychol Sci, 21, 1006.

As we walk through our environment, our visual world flows past, providing a rich source of information about the direction we are headed in. For example, imagine you are hurrying to exit an auditorium through a particular door. As you move toward your goal, your view of the textured features of the room - the floor, ceiling, and seating - move by in a very particular pattern that is specific to the direction in which you are heading. Bruggeman and Warren found that optic flow is used to calibrate the direction of movement when walking to a target, but not when throwing or kicking a ball to the same target. During an adaptation phase, participants were asked to walk to targets in a textured virtual environment in which the simulated direction of travel was $10^{\circ}$ to the left or right of the actual direction of walking. Participants readily adapted to the displaced optic flow patterns by recalibrating their initial walking direction. During pretest and posttest, participants were presented with normal optic flow containing no displacement and were asked either to throw or kick a ball to a target or to initiate walking to the target. For all three motor tasks at pretest, the directions of walking, throwing, and kicking were quite accurate. At posttest, however, walking direction showed a clear adaptation aftereffect; participants initially walked in the direction opposite the adapted optic flow displacement, reflecting their learning during the adaptation phase. Despite this evidence for the recalibration of walking, the participants showed no adaptation aftereffect for kicking and throwing. The adaptation to walking direction did not appear to alter the visual perception of "straight ahead," but rather recalibrated the mapping between the visual and the locomotor direction. Perceptual-motor calibration based on optic flow was functionally task-specific, transferring to locomotor but not to other types of motor tasks. - L.C.N.

\section{TIME PERCEPTION}

\section{The Limitations of Precise Timing Judgments}

Brenner \& Smeets (2010). How well can people judge when something happened? Vis Res, 50, 1101.

Why is it that when we have to make explicit simultaneity or temporal order judgments based on visual information, we perform rather poorly, but with interception tasks in which we have to act on a moving object, such as when we have to hit a moving ball with a bat, we are quite good? Brenner and Smeets explored this question in a series of experiments. One possible answer is that high-precision temporal judgments are obtained when observers can utilize motion information, for instance when judging the direction of apparent motion rather than the temporal order or judging the absence of motion signals rather than simultaneity. This possibility is supported by the fact that temporal order judgments are considerably better when the separation between the stimuli is small, allowing for better judgment of motion direction. If this possibility is viable, judgments of simultaneity should be interrupted by the presence of irrelevant motion. In their first experiment, Brenner and Smeets found that irrelevant motion disrupted observers' ability to match a color change occurring in two objects, and this harmful effect increased with increasing velocity.

Another possibility is that in interception tasks, observers can predict the exact moment of interception because they constantly see the moving object as it approaches the point of contact, but in typical tasks of explicit timing judgment, the stimuli are brief and involve an abrupt change that does not allow for such prediction. Brenner and Smeets's second experiment tested whether the ability to make precise timing judgmentsin order to synchronize the color change of two objects-depends on the ability to anticipate when the change would take place. Observers saw two or three rectangles that seemed to rotate in depth in various directions. The rectangles changed color at some point in time, and the observers were asked to synchronize that color change. In some of the conditions, the moment of the change was predictable - it always occurred at a specific degree of rotation (e.g., always occurred when the rectangles were orthogonal to the screen). In the other conditions, the angle at which the color change occurred varied in either direction along the path of rotation. The findings, however, did not support the hypothesis that predictability is an important factor for timing judgment. The ability of the observers to synchronize the color change was not better when the angle of rotation at the time of change was constant; instead, performance was optimal when the rectangles changed color when oriented in the frontal plane, suggesting that performance was mediated by apparent motion, because in this condition the rectangles' images were largest at the moment of change and their edges were moving most slowly.

A third and final experiment confirmed that it is hard to make precise timing judgments for stimuli that have different durations but similar intensities. The outcomes of the experiments led Brenner and Smeets to suggest that we do not have specialized mechanisms for explicit tem- 
poral judgments - perhaps because such judgments are not useful in our everyday experience-but that such specialized mechanisms do exist for motion judgments. Thus, a high level of performance in tasks that seem to require explicit timing judgments may not reflect high ability to determine when an event occurs, but may instead be due to other factors, such as motion processing. - Y.Y.

\section{SPATIAL CUING}

\section{Spatial Cuing Effects Precede Binocular Combination}

Self \& Roelfsema (2010). A monocular, unconscious form of visual attention. JVis, 10(4): Art. 17. doi:10.1167/10.4.17

Paradigms for quantifying attention abound, but one of the most popular remains the effect of a spatial precue on RTs for identifying subsequently presented objects. This paradigm was pioneered by Posner (1980, QJEP $32: 3)$. It was then refined by Jonides (1981, pp. 187ff in Long \& Baddeley, Eds., Attention and Performance IX. Hillsdale, NJ: Erlbaum), who demonstrated that an "exogenous" subset of these cues could be just as effective when they contained no information about subsequent target position. Now, in a series of clever experiments, Self and Roelfsema demonstrate that an indisputably monocular mechanism is partly responsible for exogenous cuing effects. Perhaps even more astonishing than the very early (possibly even precortical) locus for these effects is the fact that most of Self and Roelfsema's observers were completely unable to discriminate monocularly cued targets from dichoptically cued targets, despite the fact that they could identify the former targets more quickly!

Also highly recommended is Self and Roelfsema's discussion, because they do not adopt the common assumption that cuing effects reflect the benefit of attention, or of any other limited resource, for visual processing. They recognize that cues can both arouse an observer (thereby reducing
RTs) and delay his or her ability to process targets in uncued positions (and uncued eyes). Thus, an inverse relationship between the number of spatial cues and performance with cued targets might be consistent with a limited resource for attentional benefits, but it is also consistent with an unlimited resource for cuing costs. Self and Roelfsema argue strongly for the latter scenario, because they ran so many different conditions (e.g., monocular cued, monocular uncued, dichoptic cued, etc.) that they did not need to rely on trials without cues to fully constrain a linear model of costs and benefits. - J.A.S.

\section{BINOCULAR RIVALRY}

\section{Reciprocal Inhibition Confirmed}

Alais ET AL. (2010). Visual sensitivity underlying changes in visual consciousness. Curr Biol, 20, 1362.

The phenomenon of binocular rivalry has been undergoing a surge in popularity because of its use as a tool to study visual consciousness. If one eye views one stimulus while the other views another stimulus in the same location, the usual result is a perceived alternation between the two monocular images. The contents of consciousness change, while the visual inputs remain constant. How does such rivalry work, and what is the switch that produces the flip in perception? As long ago as 1901, McDougall (Brain 24:577) proposed that the two monocular images inhibit each other. At any given moment, the stronger of the two images is dominant in perception while the other image is suppressed. But why do the images alternate? If one is stronger, why isn't it continuously dominant? A standard solution to this problem is to propose some sort of adaptation that weakens the dominant stimulus, while the suppressed stimulus recovers from the adaptation during its own previous period of dominance. At some point, the suppressed stimulus becomes stronger than the dominant stimulus, and dominance flips.
The reciprocal inhibition idea can be tested by comparing the detection thresholds for probes shown to the dominant or the suppressed eye. In principle, as adaptation builds up in the dominant eye, sensitivity to probes should decline, while sensitivity should improve in the suppressed eye as it recovers from adaptation. This is a fine theory, but proving it has been difficult, because rivalry alternations are stochastic: Experimenters need to know the sensitivity just before a reversal, but they don't know exactly when a reversal is going to happen, and so can't put a probe at exactly the right point in time. Clear evidence for reciprocal inhibition has thus been lacking - until now.

In a recent article, Alais et al. argue that they have finally figured out how to do the experiment correctly. They simply had observers monitor dominance and suppression continuously and, at the same time, report whether a probe (marked by a tone) appeared in the upper or lower portion of the display. The probes appeared every few seconds. Alais et al. made the assumption that rivalry switches occurred about $450 \mathrm{msec}$ before they were reported; given that assumption, each probe could be assigned to a dominant or suppressed eye, and the timing of each probe could be assigned a relative position within a dominance epoch. That is, a given probe could be determined as having been presented to the left eye while it was suppressed and, better yet, could be localized as occurring, say, $60 \%$ of the way between the last switch in dominance and the next switch.

Once the data were sorted in this manner, the results were elegantly clear: At the beginning of an epoch, observers were $90 \%$ correct with dominant eye probes and less than $70 \%$ correct with suppressed probes. By the time dominance was about to switch, $90 \%$ of the way through the epoch, the observers were $80 \%$ correct with probes in either eye. The two eyes were then equal and, with the suppressed eye still improving, the dominance reversed. The story of 
this finding has a physics-like appeal, with its drama of a longstanding theory predicting a result whose verification waits for the right experiment. Unlike modern physics, however, we didn't need a new, expensive machine, just a clever idea. - J.M.W.

\section{VISUAL PROCESSING}

\section{High-Order Statistics of Natural Images}

TKAČIK ET AL. (in press). Local statistics in natural scenes predict the saliency of synthetic textures. PNAS.

Over the past two decades, a great deal of work has attempted to understand human vision as an adaptation specifically tailored for processing natural images. A striking new addition to this literature is provided by Tkačik et al., whose work analyzes high-order image statistics. For an organism adapted specifically to the natural world, only those high-order statistics that vary across scenes are worth sensing. The question is, which high-order statistics show significant variation across natural scenes, and which do not? In the first part of the article, the authors ask which statistics must be sensed in order to capture the local intensity histogram of a patch of a natural image. Remarkably, three statistics sufficein fact, the same three statistics that studies have revealed humans to be sensitive to when discriminating randomly scrambled mixtures of different intensities: the mean, the variance, and a third statistic called "blackshot" (Chubb et al., 2004, Vis Res 44:3223) that reflects the proportion of pixels in an image that are extremely black. In the second part of their study, the authors ask which fourth-order interpixel correlations show variation across natural images. Previous research has revealed that human observers are sensitive to fourth-order correlations between pixel intensities for some configurations of pixels but not for others (Victor \& Conte, 1991, Vis Res 31:1457). Once more, Tkačik et al. show that in natural images, fourth-order sta- tistics vary only for those pixel configurations to which human observers have previously been shown to be sensitive! - C.F.C.

\section{ATTENTIONAL CONTROL}

\section{A Limitation of Attentional Control Settings}

Al-Aidroos ET AL. (2010). You can't stop new motion: Attentional capture despite a control set for colour. Vis Cogn, 18, 859.

One of the most hotly contested issues in cognitive science over the past two decades concerns whether the control of attention can ever be purely stimulus driven-that is, whether the current focus of attention can ever be "captured" independent of the current goals and intentions of the observer. According to the contingent involuntary orienting hypothesis, originally proposed by Folk et al. (1992, JEP:HPP $18: 1030$ ), the orientation of attention is always contingent on "attentional set," which constitutes the current behavioral goals of the observer. In this view, attention may be captured by objects and events, but only when they are congruent with the current attentional set of the observer. There is now considerable empirical support for this hypothesis, but the question remains whether certain object properties might capture the focus of attention in a purely stimulus-driven fashion. Recently, evidence has accumulated to suggest that one or two special features - for example, the sudden onset of motion - might capture attention in such a fashion. However, much of this evidence has been obtained without clear control of the observer's attentional set.

To remedy this potential flaw, AlAidroos et al. examined whether an irrelevant onset-of-motion cue would capture attention even when observers were set for color. Previous evidence from Folk et al. had suggested that other features, such as the sudden appearance of an object, may capture attention when observers are set for abrupt onsets, but not when they are set for color; the onset of motion thus may also lose its ability to capture attention when observers are set for color. In Al-Aidroos et al.'s Experiment 1 , observers monitored two triangles that each appeared inside a box on either side of fixation, with the task of reporting the location of the triangle that changed color. Of critical importance, just prior to this target-defining color change, an irrelevant spatial cue was presented: In the irrelevant color cue condition, the surrounding box changed color, and in the irrelevant onset-of-motion cue condition, the box suddenly started rotating. If attentional capture was contingent on attentional set, latencies to localize the target should have been faster when the target appeared at the cued rather than the uncued location, but only when the color cue was shown. Contrary to this expectation, the findings showed that both color and onset-of-motion cues captured attention, suggesting that attentional capture by onset-of-motion cues can occur independent of attentional set.

Could these findings be attributed to some other, more general, attentional set? For instance, perhaps observers were set not for color, but for change. If so, then both the color and onset-of-motion cues would have been consistent with this set. To rule out this possibility, Al-Aidroos et al. devised a "go/no-go" task in which the triangle changed into one of two colors on each trial. One of the two colors was a signal to respond (i.e., "go"), whereas the other was a signal to withhold response (i.e., "no go"). Under these conditions, observers should no longer have been set for change, because the change associated with the no-go color was not relevant to response. In addition, color cues associated with each of these response options were presented along with the onset-of-motion cues. As expected, the results showed that the "go" color cue captured attention, but the "no-go" color cue did not. More importantly, the onset-of-motion cue also captured attention. Altogether, these findings suggest that onset of 
motion may indeed be one of the few object properties capable of controlling attention in a purely stimulusdriven fashion. - B.S.G.

\section{PERCEPTUAL LATENCY}

\section{Being Prepared to Detect}

SEIFRIED ET AL. (in press). Temporal preparation decreases perceptual latency. QJEP.

We react faster to the arrival of a signal if we know in advance when that signal is likely to occur-in other words, we benefit from temporal preparation. There is a general consensus in the information-processing literature that the effects of temporal preparation are mainly limited to late stages of processing. In a series of four experiments, Seifried et al. used a new approach to address the temporal preparation issue. Their participants watched a revolving clock hand and had to report the position of the hand at the moment a target tone was presented. The tone was soft or loud and presented under high or low temporal preparation. The index of perceptual latency was the deviation of the reported clock hand position from the actual position. In addition to decreased latencies with louder tones, Seifried et al. observed that temporal preparation decreased perceptual latency, especially in the soft-tone condition. In other words, their experiments show that temporal preparation diminishes the duration of perceptual processing and that this preparation effect is not limited to late stages of information processing. - S.G.

\section{VISUAL ATTENTION}

\section{Learning During Search Does Not Require Visual Working Memory}

VICKERY ET AL. (in press). Spatial context learning survives interference from working memory load. JEP:HPP.

Visual attention is smart, capable of using the repeated spatial layout of a display to find a target, in a phenomenon referred to as contextual cuing. Contextual cuing depends on the spatial layout of the target and distractors in a search array, independent of the identity of the distractors. Although contextual cuing indicates that attention is sensitive to learned spatial structure, the exact causes of these findings remains open. In a series of experiments, Vickery et al. asked whether visual working memory (VWM) underlies contextual cuing. VWM is a plausible candidate process because (1) several models of visual search propose that VWM holds an image of the target being searched for; (2) VWM for objects can slow attentional deployment overall; and (3) VWM for locations can slow search rates (i.e., search slopes).

Vickery et al. first examined the effect of an object VWM load on contextual cuing. During a training phase, participants were shown a memory array of four objects to either remember or ignore. Next, a visual search array appeared and participants searched for a target. In the training phase, the search displays re- peated; half of the repeated displays were paired with a VWM load, the other half were not. After the training phase, participants received a test phase that presented repeated arrays (those from the training phase) and new arrays that had not previously been seen. The results from this initial experiment showed that a VWM load slows overall search during training but does not affect learning. That is, repeated arrays paired with a memory load were learned just as quickly as those paired with no memory load: At test, all of the repeated arrays produced faster search response times than did the new, unfamiliar arrays. These findings suggest that contextual cuing does not depend on VWM.

In subsequent experiments, Vickery et al. examined different amounts of object VWM load, the effects of spatial VWM load, and the effects of the number targets for which participants searched. The results from these experiments all converged on the basic conclusion that VWM-tapped through a variety of manipulations is not necessary to produce contextual cuing in visual search. These findings add to a growing body of evidence that attention can be affected by implicit learning or priming. Vickery et al.'s article also includes results and discussion that distinguish effects on learning itself from effects on the expression of learning. These data constrain the potential mechanisms of contextual cuing, specifically, and of visual search and visual attention more generally. - S.P.V. 